\title{
Variaciones del estado cognitivo en el puerperio y sus determinantes: una revisión narrativa
}

\author{
Variations of the cognitive status in the puerperium and their \\ determinants: a narrative review
}

Eugenia Carrizo (https://orcid.org/0000-0002-5194-5261) ${ }^{1}$

Julia Domini (https://orcid.org/0000-0002-5365-023X) ${ }^{1}$

Ruth Yohana Julieta Quezada (https://orcid.org/0000-0001-5231-5029) ${ }^{1}$

Silvana Valeria Serra (https://orcid.org/0000-0003-3699-9437) ${ }^{1}$

Elio Andrés Soria (https://orcid.org/0000-0003-2373-2493) ${ }^{2}$

Agustín Ramiro Miranda (https://orcid.org/0000-0001-6673-606X) ${ }^{1}$

${ }^{1}$ Escuela de Fonoaudiología, Facultad de Ciencias Médicas, Universidad Nacional de Córdoba. Av. La Reforma, Ciudad Universitaria. 5014 Córdoba Argentina. eugenia.carrizo@

fcm.unc.edu.ar

${ }^{2}$ Consejo Nacional de Investigaciones Científicas

y Técnicas. Córdoba

Argentina.

\begin{abstract}
The neurobiological modifications that women experience during the puerperium, together with the consequent psychosocial changes, lead to variations in cognitive functions. In order to describe the cognitive variations that occur during postpartum and the determining factors, a narrative review was conducted by means of a bibliographic search in PubMed and Google Scholar. A steady increase in the number of published works was located (PubMed = 186; Google Scholar =26,730). The analysis of the articles made it possible to: a) characterize the cognitive functions during the puerperium; $b$ ) analyze the neuropsychological effects produced by the endocrinological and anatomophysiological changes; c) analyze the effect of the quality of sleep on cognition; d) analyze cognitive functions according to obstetric experiences. In conclusion, puerperal women are characterized by variations in their cognitive functions, which are determined by structural, functional, psychological and social changes. It is necessary to conduct neuropsychological research in this population, since postpartum care still focuses on the care of the newborn, without addressing the puerperal woman in a comprehensive manner.

Key words Women's health, Cognition, Postpartum Period, Neuropsychology, Parity
\end{abstract}

Resumen Las modificaciones neurobiológicas que experimenta la mujer durante el puerperio, junto con los consecuentes cambios psicosociales, determinan variaciones en el funcionamiento cognitivo. Con el objetivo de describir las variaciones cognitivas que ocurren durante el postparto y sus factores determinantes, se desarrolló una revisión narrativa a partir de la búsqueda bibliográfica en PubMed y Google Scholar. Se evidenció un constante crecimiento del número de publicaciones a lo largo de los años (PubMed=186; Google Scholar $=26730$ ). El análisis de los artículos permitió: a) Caracterizar las funciones cognitivas durante el puerperio; b) Analizar los efectos neuropsicológicos producidos por los cambios endocrinológicos y anatomofisiológicos; c) Analizar el efecto de la calidad del sueño sobre la cognición; d) Analizar las funciones cognitivas en función de las experiencias obstétricas. En conclusión, la mujer puérpera se caracteriza por presentar variaciones en sus funciones cognitivas, las cuales están determinadas por cambios estructurales, funcionales, psicológicos y sociales. Es necesario fomentar investigaciones neuropsicológicas en esta población, ya que la atención del posparto todavía se centra en el cuidado del recién nacido, sin abordar a la mujer puérpera de manera integral.

Palabras clave Salud de la Mujer, Cognición, Periodo Posparto, Neuropsicología, Paridad 


\section{Introducción}

El parto marca el comienzo de muchos cambios a largo plazo en la vida de una mujer. Sus relaciones, trabajo, actividades sociales y domésticas, y funcionamiento biológico pueden modificarse. Las mujeres durante los primeros seis meses de amamantamiento tienen una demanda psicosocial, ya sea debido a su rol primario en interacción con el infante o como consecuencia de las condiciones ambientales y sociales asociadas a dicho oll $^{1}$.

En el puerperio, la mujer se expone a las modificaciones hormonales más importantes de su vida reproductiva, caracterizado principalmente por cambios en las hormonas esteroideas y peptídicas ${ }^{2}$. Además, se establece una remodelación en la anatomofisiología de diferentes estructuras implicadas en la inducción del comportamiento materno ${ }^{3}$, la cual persiste durante al menos dos años, y tiene fines adaptativos, ayudando a las mujeres a adaptarse a la maternidad y responder a las necesidades de sus hijos ${ }^{4}$. Además, la remodelación desencadena en cambios en la cognición, el sueño, el humor, las emociones, y el comportamiento.

Una importante cantidad de literatura refiere que las hormonas que participan en el establecimiento y mantenimiento del embarazo, tienen influencia en funciones cognitivas ${ }^{2,5-7}$, y se han asociado variaciones específicas: atención, memoria, aprendizaje verbal, discriminación de respuestas relevantes e irrelevantes, habilidades visoespaciales y velocidad de procesamiento.

El presente estudio profundizará en los efectos cognitivos del puerperio teniendo en cuenta factores influyentes, con el fin de dilucidar cuál es la modulación en el funcionamiento cognitivo de la mujer puérpera.

\section{Metodología}

Se realizó una revisión narrativa de literatura. Estos artículos son útiles para describir y discutir el estado del arte en un determinado asunto, posibilitando una discusión ampliada ${ }^{8}$. Los artículos en idioma inglés y español fueron identificados por búsqueda bibliográfica en PubMed (National Library of Medicine of the United States) y Google Scholar. Las búsquedas se basaron en la pregunta de investigación: ¿Cuáles son las variaciones cognitivas que ocurren durante el periodo de postparto en mujeres y cuáles son sus posibles determinantes? Con el fin de evaluar la asociación del puerperio y la cognición, se utilizaron como descriptores: ("Postpartum” OR "Puerperium”) AND (“Cognition" OR “Cognitive functions"). La búsqueda se realizó sin limitación de fecha, país de desarrollo o área de conocimiento. Se incluyeron artículos originales, de revisión, y otros tipos de publicaciones (resúmenes publicados en revistas y tesis). Los resultados se presentan en cuatro secciones a los fines de responder la pregunta de investigación planteada.

\section{Resultados y discusión}

En la Figura 1 se presenta la evolución temporal de cantidad de artículos publicados disponibles en PubMed y Google Scholar. Se puede observar un crecimiento exponencial del número de artículos acerca de la temática planteada, con una pendiente más pronunciada en los últimos 7 años. La búsqueda en PubMed arrojó una cifra de 186 artículos, mientras que la utilización de los mismos descriptores en Google Scholar se obtuvo 26.730 artículos. Se seleccionaron 39 artículos (Cuadro 1) según los criterios de inclusión: temática específica, menos de 20 años de antigüedad, relevancia al documentar, reseñar o representar específicamente las variaciones cognitivas durante el puerperio. Estos artículos fueron revisiones $(n=10)$, estudios longitudinales $(n=12)$, estudios transversales $(n=6)$, y estudios pre-clínicos experimentales $(n=11)$. Luego se identificaron, clasificaron y analizaron de acuerdo con los planteamientos sobre el tema, lo cual permitió definir las categorías de presentación.

\section{Caracterización cognitiva del período postparto}

Durante el postparto un gran número de mujeres experimenta cambios cognitivos asociados al maternal brain ${ }^{9}$. Las investigaciones sugieren que hay variaciones cognitivas específicas asociadas con el embarazo, y, en general, el embarazo ha recibido más atención que el período postparto en el ámbito de la investigación ${ }^{3}$.

Los estudios de desempeño cognitivo en mujeres embarazadas consistentemente hallaron que la mayoría de las mujeres reportan declive cognitivo, especialmente en la memoria ${ }^{10}$. Las quejas más reportadas son olvidos y disturbios mnésicos, pobre concentración, aumento de la distracción y dificultad lectora. Los déficits cognitivos comúnmente asociados al embarazo y postparto se refieren a déficit atencional, memoria visual, dificultad con el aprendizaje verbal, discrimina- 


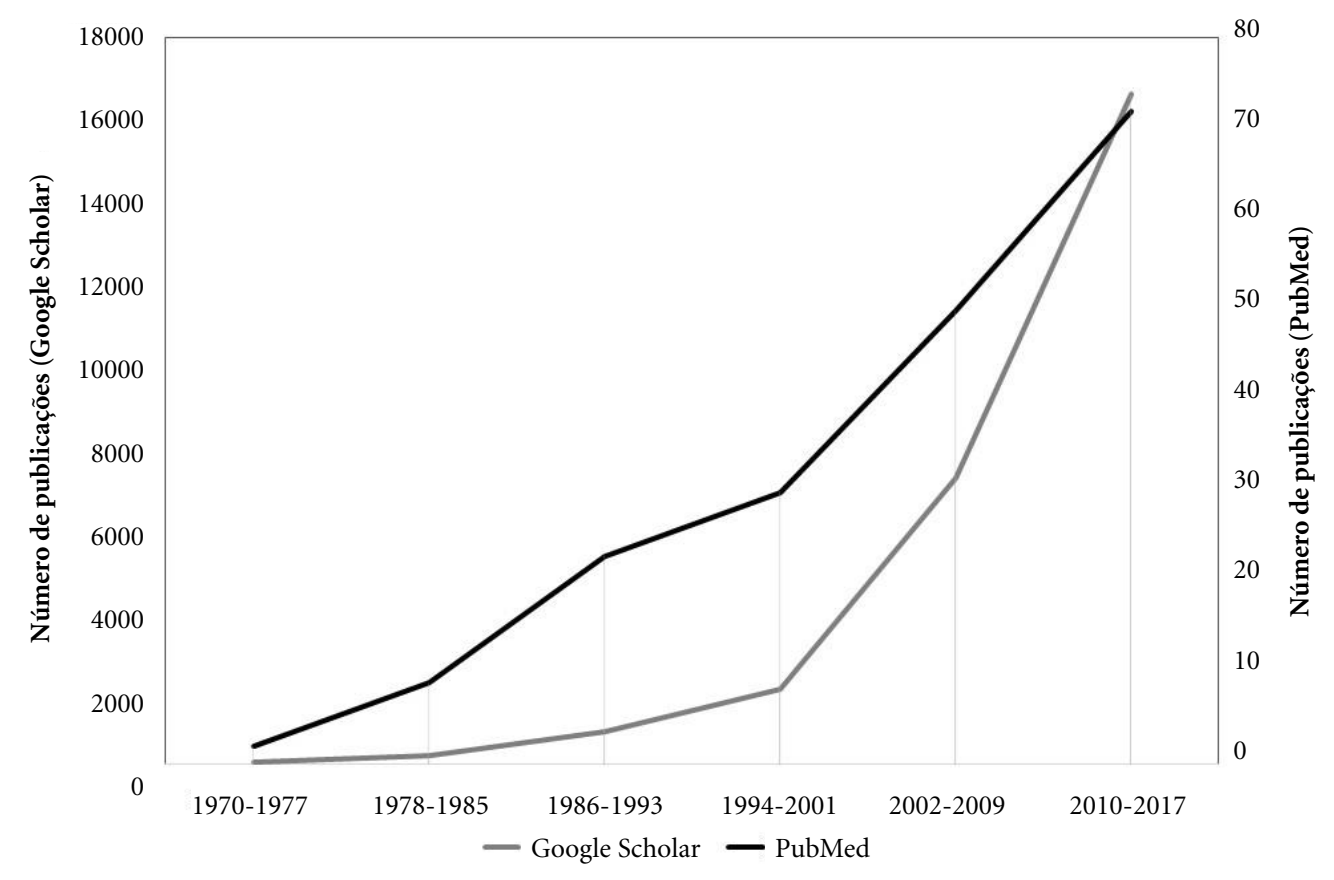

Figura 1. Número de publicaciones encontradas para la estrategia ("Postpartum" OR "Puerperium") AND (“Cognition” OR “Cognitive functions”) en PubMed y Google Scholar.

ción de respuestas relevantes e irrelevantes, habilidad espacial ${ }^{2}$, memoria verbal ${ }^{2,4,11}$ y velocidad de procesamiento $^{2,12}$.

Respecto a los déficits de memoria, al menos el 50\% de las mujeres reportan cambios durante estos períodos, usualmente olvidos de la rutina diaria, lo cuales pueden extenderse hasta dos meses o más después del parto ${ }^{10,13}$, o incluso hasta tres meses postparto para la memoria de recuerdo verbal ${ }^{11}$.

Múltiples estudios reportan un menor desempeño en la memoria implícita, explícita, prospectiva, listas de aprendizaje, y memoria verbal en pruebas a mujeres embarazadas comparados con mujeres no embarazadas/nulíparas ${ }^{2,14-17}$. Aunque existan quejas subjetivas, no siempre son correlativas a las mediciones proporcionadas por test objetivos ${ }^{10,14}$. Esto marca una controversia entre los resultados obtenidos por diferentes investigadores.

Casey et al. ${ }^{18}$ con el objetivo de investigar cambios mnésicos, aplicaron una batería de test de memoria a 111 mujeres embarazadas primigrávidas/multigrávidas, puérperas y controles. Concluyeron en que si había una disminución en la capacidad de la memoria, solo era el área de la memoria de trabajo, que incrementaría los olvidos de la rutina diaria y errores para llevar a cabo varias pruebas mentales al mismo tiempo; lo que conduciría a los problemas reportados de memoria. Los autores confirmaron que muchas mujeres embarazadas experimentan cambios cognitivos, pero que pueden llevar a cabo demandas atencionales en pruebas requeridas. Adhirieron a esto Janes et al. ${ }^{19}$, quienes concluyeron que las mujeres puntúan más bajo que los controles en pruebas de memoria de trabajo ${ }^{13}$.

De Groot et al. ${ }^{16}$ refirieron que la codificación y recuperación de la memoria parecía estar comprometidas en mujeres embarazadas en relación con mujeres no embarazadas, y esta diferencia se encontraba todavía 32 semanas después del parto. Por lo tanto, el rendimiento de la memoria es menor durante el embarazo y la maternidad temprana. La diferencia que se pudo establecer entre el embarazo y el puerperio, fue que esta etapa además del déficit de memoria se caracteriza por la presencia lentitud en el procesamiento de la información. Messinis et al. ${ }^{20}$ examinaron el funcionamiento cognitivo en puérperas con 
Cuadro 1. Principales características de los 39 estudios incluidos en la revisión.

\begin{tabular}{|c|c|c|}
\hline Autor (año) & Diseño & Objetivo \\
\hline $\begin{array}{l}\text { Henry y } \\
\text { Sherwin } \\
(2012)^{2}\end{array}$ & Longitudinal & $\begin{array}{l}\text { Analizar la influencia del estradiol, la progesterona, la testosterona, niveles de cortisol } \\
\text { y prolactina en el funcionamiento cognitivo durante el final del embarazo y el } \\
\text { período postparto temprano. }\end{array}$ \\
\hline $\begin{array}{l}\text { Workman et } \\
\text { al. }(2012)^{3}\end{array}$ & Revisión & $\begin{array}{l}\text { Presentar literatura sobre los cambios relacionados con el embarazo y la paridad } \\
\text { tanto en la cognición como en el afecto y cómo estos cambios probablemente } \\
\text { involucren cambios plásticos dentro del hipocampo, una región que es sensible a las } \\
\text { hormonas reproductivas. }\end{array}$ \\
\hline $\begin{array}{l}\text { Hoekzema et } \\
\text { al. }(2016)^{4}\end{array}$ & Longitudinal & $\begin{array}{l}\text { Estudiar los cambios en la estructura de la sustancia gris de mujeres primerizas y } \\
\text { nulíparas. }\end{array}$ \\
\hline $\begin{array}{l}\text { Barha y Galea } \\
(2010)^{6}\end{array}$ & Revisión & $\begin{array}{l}\text { Revisar la literatura sobre los efectos estrogénicos en la neurogénesis y plasticidad } \\
\text { sináptica en el hipocampo, así como en la cognición. }\end{array}$ \\
\hline $\begin{array}{l}\text { Kim et al. } \\
(2010)^{9}\end{array}$ & Longitudinal & $\begin{array}{l}\text { Examinar los cambios de la sustancia gris utilizando morfometría basada en vóxeles } \\
\text { en imágenes de resonancia magnética de alta resolución de los cerebros de mujeres } \\
\text { en dos momentos: } 2-4 \text { semanas y 3-4 meses después del parto. }\end{array}$ \\
\hline $\begin{array}{l}\text { Logan et al. } \\
(2014)^{10}\end{array}$ & Longitudinal & $\begin{array}{l}\text { 1. Determinar diferencias longitudinales de grupo en las medidas de funcionamiento } \\
\text { cognitivo (memoria, la atención, la función ejecutiva y el razonamiento } \\
\text { visuoespacial) entre mujeres embarazadas/posparto y no embarazadas/nulíparas. } \\
\text { 2. Evaluar el potencial papel moderador del estado de ánimo y el bienestar subjetivo } \\
\text { (incluida la calidad de vida) sobre el funcionamiento neuropsicológico durante el } \\
\text { embarazo. }\end{array}$ \\
\hline $\begin{array}{l}\text { Glynn } \\
(2010)^{11}\end{array}$ & Longitudinal & $\begin{array}{l}\text { 1. Documentar los cambios en el rendimiento de la memoria durante el embarazo y } \\
\text { el posparto temprano. } \\
\text { 2. Evaluar la asociación entre las exposiciones endocrinas durante la gestación y los } \\
\text { cambios en la función de la memoria exhibidos por las mujeres embarazadas. }\end{array}$ \\
\hline $\begin{array}{l}\text { Christensen } \\
\text { et al. }(2010)^{12}\end{array}$ & Longitudinal & $\begin{array}{l}\text { Determinar si el embarazo y la maternidad están asociados con un cambio cognitivo } \\
\text { a corto o largo plazo midiendo la cognición durante y antes del embarazo y la } \\
\text { maternidad. }\end{array}$ \\
\hline $\begin{array}{l}\text { Anderson y } \\
\text { Rutherford } \\
(2012)^{13}\end{array}$ & Revisión & $\begin{array}{l}\text { Examinar la reorganización cognitiva como el resultado de la experiencia } \\
\text { reproductiva desde una perspectiva adaptacionista. }\end{array}$ \\
\hline $\begin{array}{l}\text { Cuttler et al. } \\
(2011)^{14}\end{array}$ & Transversal & $\begin{array}{l}\text { Analizar las causas para la discrepancia entre los hallazgos de los déficits de memoria } \\
\text { subjetivos y objetivos, así como las fuentes de quejas de memoria de las mujeres } \\
\text { embarazadas. }\end{array}$ \\
\hline $\begin{array}{l}\text { de Groot et al. } \\
(2006)^{16}\end{array}$ & Longitudinal & $\begin{array}{l}\text { 1. Examinar las diferencias en el rendimiento de una batería neurocognitiva entre } \\
\text { mujeres embarazadas y mujeres en un grupo de control. } \\
\text { 2. Analizar si las funciones cognitivas se vieron afectadas de forma diferente durante } \\
\text { las diversas etapas del embarazo y el período posparto. }\end{array}$ \\
\hline $\begin{array}{l}\text { de Groot et al. } \\
(2003)^{17}\end{array}$ & Longitudinal & $\begin{array}{l}\text { 1. Determinar si la atención selectiva es diferente entre las mujeres embarazadas y las } \\
\text { mujeres no embarazadas emparejadas por edad y educación. } \\
\text { 2. Analizar diferencias en la atención selectiva entre los dos grupos después del parto. }\end{array}$ \\
\hline $\begin{array}{l}\text { Cassey et al. } \\
(1999)^{18}\end{array}$ & Transversal & $\begin{array}{l}\text { 1. Estudiar los cambios en la memoria implícita en el embarazo. } \\
\text { 2. Emplear un conjunto de pruebas objetivas para la identificación alteraciones de } \\
\text { memoria en el embarazo o postparto. } \\
\text { 3. Probar la hipótesis de que el cambio en el patrón de sueño informado es un } \\
\text { predictor significativo del cambio en la memoria reportada. }\end{array}$ \\
\hline $\begin{array}{l}\text { Janes et al. } \\
(1999)^{19}\end{array}$ & Transversal & $\begin{array}{l}\text { Comparar los rendimientos de la memoria tanto objetiva como reportada durante el } \\
\text { embarazo y puerperio en mujeres primigrávida, primíparas y nulíparas, emparejadas } \\
\text { en edad y niveles de educación. }\end{array}$ \\
\hline $\begin{array}{l}\text { Messinis et al. } \\
(2010)^{20}\end{array}$ & Transversal & $\begin{array}{l}\text { Examinar el funcionamiento cognitivo en mujeres puérperas sin depresión posparto } \\
\text { versus puérperas con depresión, y comparar su desempeño con el de controles } \\
\text { femeninos no deprimidos y no puérperas. }\end{array}$ \\
\hline $\begin{array}{l}\text { Buckwalter et } \\
\text { al. }(2001)^{21}\end{array}$ & Revisión & $\begin{array}{l}\text { Proporcionar una revisión sobre la neuroactividad de los esteroides, y evidenciar } \\
\text { los paradigmas observacionales y experimentales que relacionan las hormonas y los } \\
\text { aspectos clínicos de la cognición y el estado de ánimo en los humanos. }\end{array}$ \\
\hline
\end{tabular}


Cuadro 1. Principales características de los 39 estudios incluidos en la revisión.

\begin{tabular}{|c|c|c|}
\hline Autor (año) & Diseño & Objetivo \\
\hline $\begin{array}{l}\text { Crawley et al. } \\
(2003)^{22}\end{array}$ & Longitudinal & $\begin{array}{l}\text { Examinar el rendimiento objetivo y la autopercepción de la función cognitiva } \\
\text { durante el embarazo y el período postparto. }\end{array}$ \\
\hline $\begin{array}{l}\text { Brett y } \\
\text { Baxendale } \\
(2001)^{23}\end{array}$ & Revisión & Revisar la literatura sobre el cambio cognitivo durante y después del embarazo. \\
\hline $\begin{array}{l}\text { Numan y } \\
\text { Woodside } \\
(2010)^{24}\end{array}$ & Revisión & $\begin{array}{l}\text { Revisar la literatura sobre el sustrato neuronal de la capacidad de respuesta materna } \\
\text { y sobre las adaptaciones fisiológicas, de comportamiento y de motivación al estado } \\
\text { materno. }\end{array}$ \\
\hline $\begin{array}{l}\text { Numan } \\
(2006)^{25}\end{array}$ & Revisión & $\begin{array}{l}\text { Revisar la regulación neuronal y neuroquímica del comportamiento materno, } \\
\text { enfatizando el papel del área preóptica medial y sus conexiones neuronales. }\end{array}$ \\
\hline $\begin{array}{l}\text { Scanlan et al. } \\
(2006)^{26}\end{array}$ & $\begin{array}{l}\text { Pre-clínico } \\
\text { Experimental }\end{array}$ & $\begin{array}{l}\text { Analizar la memoria materna en ratas nulíparas y primíparas, evaluando el efecto de } \\
55 \text { o más días de aislamiento de sus crías. }\end{array}$ \\
\hline $\begin{array}{l}\text { Stolzenberg et } \\
\text { al. }(2012)^{27}\end{array}$ & $\begin{array}{l}\text { Pre-clínico } \\
\text { Experimental }\end{array}$ & Analizar los efectos epigenéticos en el comportamiento materno. \\
\hline $\begin{array}{l}\text { Parsons et al. } \\
(2004)^{28}\end{array}$ & Longitudinal & $\begin{array}{l}\text { Evaluar si el embarazo previo, que determina el ambiente hormonal posterior de una } \\
\text { mujer, se asocia con un perfil cognitivo específico durante y después del embarazo. }\end{array}$ \\
\hline $\begin{array}{l}\text { Holmes et al. } \\
(2002)^{30}\end{array}$ & $\begin{array}{l}\text { Pre-clínico } \\
\text { Experimental }\end{array}$ & $\begin{array}{l}\text { Estudiar los efectos de diferentes dosis de estradiol sobre el rendimiento en } \\
\text { habilidades visoespaciales. }\end{array}$ \\
\hline $\begin{array}{l}\text { Vanston } \\
\text { y Watson } \\
(2005)^{31}\end{array}$ & Longitudinal & $\begin{array}{l}\text { Analizar el desempeño cognitivo de mujeres desde el inicio del embarazo hasta la } \\
\text { reanudación posnatal de la menstruación. }\end{array}$ \\
\hline \begin{tabular}{l|l} 
Neumann \\
$(2003)^{42}$
\end{tabular} & Revisión & $\begin{array}{l}\text { Revisar los mecanismos cerebrales subyacentes a las alteraciones emocionales en el } \\
\text { período de postparto. }\end{array}$ \\
\hline $\begin{array}{l}\text { Oatridge et al. } \\
(2002)^{43}\end{array}$ & Longitudinal & $\begin{array}{l}\text { Evaluar cuantitativamente los cambios cerebrales durante y después del embarazo } \\
\text { saludable y comparar estos cambios con los observados en casos de preeclampsia. }\end{array}$ \\
\hline $\begin{array}{l}\text { Leuner } \\
\text { y Gould } \\
(2010)^{44}\end{array}$ & $\begin{array}{l}\text { Pre-clínico } \\
\text { Experimental }\end{array}$ & Analizar la estructura y función de la corteza durante el período posparto. \\
\hline $\begin{array}{l}\text { Macbeth } \\
\text { y Luine } \\
(2010)^{45}\end{array}$ & Revisión & $\begin{array}{l}\text { Revisar sobre los cambios en la ansiedad y la cognición en función a la experiencia } \\
\text { reproductiva. }\end{array}$ \\
\hline $\begin{array}{l}\text { Pawluski et al. } \\
(2009)^{47}\end{array}$ & Revisión & Revisar sobre las variaciones en la neurogénesis del hipocampo a lo largo de la vida. \\
\hline $\begin{array}{l}\text { Pawluski } \\
\text { y Galea } \\
(2007)^{52}\end{array}$ & $\begin{array}{l}\text { Pre-clínico } \\
\text { Experimental }\end{array}$ & $\begin{array}{l}\text { Determinar el rol de la experiencia reproductiva en la neurogénesis del hipocampo } \\
\text { a través de la proliferación y viabilidad celular, y determinar si las diferencias se } \\
\text { debieron al efecto del embarazo y/o la exposición de la cría. }\end{array}$ \\
\hline $\begin{array}{l}\text { Galea et al. } \\
(2000)^{53}\end{array}$ & $\begin{array}{l}\text { Pre-clínico } \\
\text { Experimental }\end{array}$ & Determinar el efecto del embarazo en las habilidades espaciales. \\
\hline $\begin{array}{l}\text { Bodensteiner } \\
\text { et al. }(2006)^{55}\end{array}$ & $\begin{array}{l}\text { Pre-clínico } \\
\text { Experimental }\end{array}$ & Determinar si el ambiente hormonal del embarazo afecta la cognición espacial. \\
\hline $\begin{array}{l}\text { Love et al. } \\
(2005)^{56}\end{array}$ & $\begin{array}{l}\text { Pre-clínico } \\
\text { Experimental }\end{array}$ & $\begin{array}{l}\text { Examinar la duración de los efectos neurobiológicos y comportamentales en } \\
\text { ratas hembras expuestas a las variaciones endocrinológicas que acompañan al } \\
\text { apareamiento, el embarazo, el parto y la lactancia. }\end{array}$ \\
\hline $\begin{array}{l}\text { Gatewood et } \\
\text { al. }(2005)^{57}\end{array}$ & $\begin{array}{l}\text { Pre-clínico } \\
\text { Experimental }\end{array}$ & $\begin{array}{l}\text { Analizar el aprendizaje espacial y la memoria en nulíparas, primíparas y multíparas } \\
\text { emparejadas por edad. }\end{array}$ \\
\hline $\begin{array}{l}\text { Keyser- } \\
\text { Marcus et al. } \\
(2001)^{58}\end{array}$ & $\begin{array}{l}\text { Pre-clínico } \\
\text { Experimental }\end{array}$ & Examinar los concomitantes neuroendocrinos en el área preóptica medial. \\
\hline $\begin{array}{l}\text { Paris y Frye } \\
(2008)^{59}\end{array}$ & $\begin{array}{l}\text { Pre-clínico } \\
\text { Experimental }\end{array}$ & $\begin{array}{l}\text { Abordar el rol de los factores hormonales en la mejora cognitiva asociada con la } \\
\text { paridad. }\end{array}$ \\
\hline $\begin{array}{l}\text { Carrizo et al. } \\
(2017)^{60}\end{array}$ & Transversal & $\begin{array}{l}\text { Determinar qué relación existe entre los fallos en la producción verbal dirigida y la } \\
\text { paridad, características sociosanitarias y severidad del insomnio en puérperas. }\end{array}$ \\
\hline $\begin{array}{l}\text { Miranda et al. } \\
(2017)^{61}\end{array}$ & Transversal & $\begin{array}{l}\text { Conocer y comparar las estrategias de organización de producción verbal en mujeres } \\
\text { puérperas lactantes y mujeres no puérperas, apareadas en edad y nivel educativo. }\end{array}$ \\
\hline
\end{tabular}


depresión y compararon el desempeño con un grupo de controles (no puérperas ni deprimidas) encontrándose menor rendimiento mnésico en las mujeres postparto.

Otro dominio estudiado dentro del funcionamiento cognitivo fue la atención. Buckwalter et al. ${ }^{21}$ encontraron una tendencia de las mujeres a tener menor rendimiento en la prueba de Stroop en los últimos dos meses de embarazo en comparación con dos a seis semanas después del parto $^{16}$. Por otro lado, Crawley et al. ${ }^{22}$ realizaron un estudio longitudinal en el cual se le administraron tres pruebas cognitivas a un grupo de mujeres embarazadas y no embarazadas, haciendo mediciones durante el segundo, tercer trimestre de embarazo, sexta semana postparto y un año postparto. El análisis del desempeño reveló que para todas las mujeres hubo una diferencia significativa en la prueba: el grado de interferencia para la atención enfocada fue significativamente mayor en la primera medición (segundo trimestre de embarazo) que en la cuarta (un año postparto). Los autores informaron que las mujeres embarazadas auto-reportaban que su capacidad de atención dividida, concentración y recuerdo verbal eran más bajas en el tercer trimestre de embarazo. Las embarazadas puntuaron en las escalas de autopercepción un rendimiento más bajo que previo al embarazo. Esta percepción se mantuvo estable incluso durante el primer año postparto. Los autores atribuyeron esto a una posible adaptación a esta nueva situación cognitiva.

\section{Cambios hormonales y sus implicancias en las funciones cognitivas}

El embarazo y periodo postparto están caracterizados por los cambios hormonales más dramáticos que las mujeres experimentan durante su vida reproductiva ${ }^{2}$. Los niveles de estradiol, progesterona y cortisol se incrementan en gran parte debido a las secreciones de la placenta luego de su expulsión hay rápidos decrecimientos en los niveles de hormonas esteroideas y peptídicas, generando que las mujeres sean hipogonadales por alrededor de 180 días postparto ${ }^{23}$.

Las fluctuaciones esteroideas y peptídicas están relacionadas con modificaciones anatomofuncionales en cerebro, en áreas que están implicadas en la inducción del comportamiento materno y en satisfacer las demandas de lactancia de la mujer/madre en orden de asegurar la supervivencia de su descendencia ${ }^{5}$.

Los eventos endocrinos durante el embarazo y el postparto inician la parición, el comporta- miento materno, la lactancia, la agresión y el reconocimiento de la descendencia ${ }^{24}$. En modelos animales se ha comprobado que los cambios hormonales estimulan el comportamiento maternal e inducen cambios permanentes en la sensibilidad maternal (memoria materna) ${ }^{25-27}$.

Numerosas hormonas esenciales en el establecimiento y mantenimiento del embarazo tienen influencia en funciones cognitivas ${ }^{2,5-7}$. Las hormonas esteroideas modulan el funcionamiento cognitivo en mujeres jóvenes cíclicas y en menopáusicas. Por ejemplo, hay cambios en el desempeño cognitivo en mujeres cuando están muy cerca del ciclo menstrual, desempeñándose mejor en habilidades espaciales cuando los niveles de estradiol están bajos y cuando los niveles de estradiol están altos².

Henry y Sherwin ${ }^{2}$ investigaron cambios cognitivos específicas durante el último trimestre del embarazo y postparto temprano, según los niveles de estradiol, progesterona, testosterona, cortisol y prolactina. Estas son las hormonas esteroideas comúnmente asociadas con el funcionamiento cognitivo y el estado de ánimo ${ }^{28}$. Henry y Sherwin ${ }^{2}$ compararon a un grupo de embarazadas/puérperas con un grupo de no embarazadas. Como el diseño del estudio fue de tipo longitudinal, a cada participante del grupo embarazadas/ puérperas se le realizó la misma evaluación cognitiva en el último trimestre del embarazo y en el período inicial de puerperio. Las mujeres del grupo de embarazadas/puérperas tuvieron peor rendimiento que las del grupo no embarazadas en pruebas de memoria verbal, apareamiento de palabra, aprendizaje asociativo y velocidad de procesamiento. La evidencia hasta la fecha sugiere que durante el embarazo y el postparto las mujeres pueden experimentar dificultades en la codificación y recuperación de información verbal no relacionada, pero no de información contextual ${ }^{2}$.

Además, pudieron hallar asociaciones entre los niveles hormonales y el desempeño cognitivo, relacionando: niveles de estradiol y puntajes de atención; cortisol y memoria verbal, cortisol y atención (en puérperas), cortisol y habilidades espaciales (en puérperas). Niveles más altos y más bajos de cortisol fueron asociados con un desempeño más pobre, mientras que los niveles moderados fueron óptimos y se asociaron a un mejor rendimiento. Por otro lado, niveles más bajos de estradiol han sido asociados con mejor habilidad visoespacial mientras que niveles más altos están asociados con habilidad espacial pobre ${ }^{29,30}$, por lo que las fluctuaciones en el nivel de estradiol a lo 
largo del embarazo podrían preceder a los efectos de la habilidad espacial ${ }^{5}$.

En cuanto al rol específico de la prolactina, el estudio de Henry y Sherwin ${ }^{2}$ fue el primero en encontrar una relación entre los niveles de prolactina y el funcionamiento cognitivo en mujeres jóvenes embarazadas. Hallaron que los niveles de prolactina durante el embarazo se asocian con un funcionamiento ejecutivo disminuido y menor memoria de recuerdo verbal. A pesar de que los mecanismos por los cuales la prolactina podría influenciar la función cognitiva son desconocidos, es posible especular que podría estar asociado con el efecto inhibitorio de la dopamina sobre la prolactina. En cuestión, los niveles de prolactina son tomados como un indicador indirecto de la función dopamina, dónde niveles más altos de prolactina son indicadores de niveles más bajos de dopamina y viceversa. Niveles más altos de dopamina fueron asociados con mejor rendimiento en memoria verbal y memoria de trabajo. Por lo tanto, en el embarazo, al estar muy alta la hormona prolactina, el funcionamiento de dopamina sería menor.

Otros estudios también han encontrado déficits específicos en los dominios cognitivos como la memoria de trabajo, la memoria verbal o la atención en mujeres embarazadas ${ }^{11,31}$, que también pueden estar relacionados con los niveles hormonales maternos ${ }^{5}$. Por ejemplo, en las mujeres embarazadas durante el tercer trimestre se observa un menor rendimiento en la memoria de recuerdo verbal en comparación con las mujeres no embarazadas. Este cambio está relacionado con los niveles maternos de hormonas esteroideas, ya que los niveles de cortisol fueron más bajos y los niveles de estradiol fueron más altos en mujeres embarazadas con peor rendimiento ${ }^{11}$.

Los niveles de estradiol y progesterona cambian durante el embarazo, y estas fluctuaciones pueden contribuir a las modificaciones observadas en la memoria dependiente del hipocampo en murinos y mediar potencialmente muchos de los efectos cognitivos observados con la experiencia reproductiva, ya que las fluctuaciones de estas hormonas en otros momentos de la vida afectan la cognición ${ }^{5}$.

\section{Cambios en el sueño y sus implicancias en las funciones cognitivas}

Hay un creciente interés en estudiar el rol del sueño en el funcionamiento cognitivo, así como las implicaciones de la privación de sueño para la declinación de las habilidades cognitivas, con el fin de comprender mejor la correlación entre $\operatorname{ambos}^{32}$.

Desde una perspectiva neurofisiológica, Banks y Dinges ${ }^{33}$, manifiestan que los estados de vigilia y sueño son modulados por un reloj biológico hipotálamico, que regula aspectos del comportamiento como la somnolencia y el desempeño cognitivo, generando ritmos circadianos. Los procesos homeostáticos que regulan el sueño y los ritmos circadianos determinan la estabilidad de las funciones cognitivas.

La privación del sueño puede elevar la presión homeostática al punto de que las funciones cognitivas de vigilia se degraden aún en el momento del pico del ciclo circadiano para el estado de vigilia. Esta degradación de las funciones cognitivas parece reflejar una intrusión transitoria de la neurobiología del sueño en la neurobiología de estar despierto ${ }^{34}$.

En consecuencia, la cronodisrupción puede acarrear complicaciones en la salud y el bienestar, generando sensación de malestar, fatiga, y fluctuaciones en el humor, entre otras ${ }^{35}$. Más aún, los malos hábitos del sueño se relacionan con problemas cognitivos, y capacidades mentales reducidas $^{36}$. Periodos extensos con reducidas horas de sueño y la sensación de fatiga están asociados a una disminución en la concentración y memo$\mathrm{ria}^{37}$.

La privación del sueño se asocia con importantes costos relacionados con la salud, en gran medida porque deteriora el rendimiento cognitivo debido al incremento de la propensión a dormirse y la inestabilidad de despertar las funciones neuroconductuales ${ }^{37}$. Entre las funciones cognitivas particularmente afectadas por pérdida de sueño se encuentran la velocidad psicomotora y cognitiva, la atención ejecutiva y el estado de alerta (vigilancia), la memoria de trabajo y otras habilidades más complejas como las funciones ejecutivas ${ }^{34}$. Además se generan cambios emocionales como estado de ánimo deprimido o aumento de la sensación de somnolencia y cansancio $^{33}$.

Estudios llevados a cabo por Nielson et al..$^{38}$ afirmaron menor duración y calidad de sueño puede producir importantes consecuencias en el desempeño cognitivo, ocasionado disminución de la concentración, del estado de alerta, del tiempo de reacción y del desempeño en general. Siguiendo esta línea, Alvarado et al. ${ }^{37}$ concluyeron en que la restricción o disminución en las horas efectivas de sueño es uno de los factores primordiales que explican el deterioro en el rendimiento cognitivo. 
Diversas investigaciones revelaron que frente a la privación de sueño se puede esperar: reducción de la alerta conductual y el desempeño cognitivo, ocurrencia de microsueños involuntarios, inestabilidad del desempeño en el nivel de atención que genera errores de omisión (lapsos) o de comisión (respuestas incorrectas), procesos cognitivos más lentos al realizar tareas específicas; asimismo que la presión del tiempo genera errores cognitivos, descenso de la memoria a corto plazo y la capacidad de retención, menos facilidad para realizar varias labores o tener en mente distintos pensamientos a la vez (multitasking), disminución de la memoria de trabajo, reducción de la capacidad de aprendizaje y del uso correcto del lenguaje, deterioro de los procesos de pensamiento complejo, abstracto o divergente, incremento de los errores de respuesta de supresión por la corteza prefrontal, inefectividad para resolver problemas, encontrar soluciones o apego a ideas específicas, deterioro del rendimiento de una acción llevada a cabo en el tiempo, incremento de las sensaciones de somnolencia, fatiga y confusión, disminución del vigor y la alerta e incremento de las sensaciones de irritabilidad, ansiedad y depresión ${ }^{33,34}$.

Las modificaciones en el sueño pueden estar presentes en cualquier persona afectando su funcionamiento cognitivo, incluso en mujeres durante el período posparto ${ }^{16}$. Swain et al..$^{39}$ llevaron a cabo un estudio en mujeres primerizas en su período postparto para indagar sobre los cambios de humor, sueño y funciones cognitivas. Las mujeres postparto reportaron más despertares nocturnos, más tiempo despiertas esperando dormir, y más siestas que las de un grupo control, aunque en general los tiempos de sueño fueron similares. Concluyeron además en que las mujeres deben hacer importantes ajustes en sus patrones de sueño durante el período postparto, que, en su grupo de estudio, fueron en gran parte exitosos, particularmente después de la primera semana postparto.

Bei et al..$^{40}$ afirman que hay compromisos en cuanto a la duración y calidad del sueño que son comúnmente reportados por las mujeres y confirmados por mediciones objetivas de sueño. El mal sueño informado por auto-reporte se ha asociado con alteraciones concurrentes del estado de ánimo y con un mayor riesgo de futuros problemas de humor durante el embarazo y período postparto. $\mathrm{McBean}{ }^{41}$ realizaron un estudio experimental en mujeres nulíparas, en cual se generó artificialmente un estado de fragmentación del sueño nocturno similar al que ocurre en las mujeres en periodo postparto. Los resultados obtenidos indicaron que las mujeres nulíparas tuvieron una disminución significativa de la calidad del sueño y el estado de ánimo. Por lo tanto, el período postparto, caracterizado por la fragmentación del sueño mantenida en el tiempo, se asocia con deterioro diurno, alteraciones en la salud mental, además del complejo conjunto de cambios a los que se encuentran sometidas.

\section{Cambios en la anatomía y fisiología del cerebro de las mujeres en período postparto}

Es conocido que la experiencia reproductiva (embarazo y paridad) en los mamíferos produce alteraciones en la fisiología materna, que son necesarias para mantener la gestación, el desarrollo fetal y el parto, y además sirven para asegurar la supervivencia de los hijos ${ }^{42}$. Dichas alteraciones podrían también preparar al cerebro de la mujer para enfrentar los desafíos de la maternidad, donde el cerebro podría atravesar algunos cambios y ser remodelado, proceso que se inicia antes del parto. No obstante eso, no es frecuente que una adaptación se obtenga sin un costo asociado. Para los humanos, en el caso del "nuevo" cerebro materno, la disminución de la memoria sería uno de los $\operatorname{costos}^{11}$.

En las mujeres el tamaño del cerebro decrece aproximadamente un 7\% en el curso del embarazo, alcanzando su máximo en el parto y retornando al tamaño preconcepción seis meses después del nacimiento ${ }^{43}$. Esto se acompaña de modificaciones morfológicas y fisiológicas de diversas estructuras cerebrales implicadas en la inducción del comportamiento materno ${ }^{3}$, por ejemplo el hipocampo y la corteza ${ }^{44-47}$. Además se produce una cantidad sustancial de plasticidad neuronal para preparar a la mujer ${ }^{24}$.

El hipocampo cumple una función primordial en la consolidación de la información y su almacenamiento a largo plazo ${ }^{48} \mathrm{y}$ tiene un papel importante en la mediación de numerosos tipos de aprendizaje y memoria ${ }^{49,50}$. Mantiene un alto grado de plasticidad a lo largo de la vida y contiene receptores de hormonas esteroideas. Con la experiencia reproductiva, la gran concentración de receptores de hormonas esteroideas y peptídicas aumentan la sensibilidad del hipocampo a las fluctuaciones hormonales, pudiendo ser modificado en su estructura y plasticidad ${ }^{3,47,51,52}$. Por otra parte, en cuanto a la morfología cerebral, se ha demostrado en roedores que el tamaño total del cerebro disminuye durante el embarazo y 
vuelve al tamaño preconcepcional durante el período postparto ${ }^{43,53}$.

En relación a los cambios estructurales del cerebro, las mujeres embarazadas experimentan una remodelación significativa que persiste durante al menos dos años después del parto, con fines adaptativos, ayudando a las mujeres a adaptarse a la maternidad y responder a las necesidades de sus hijos ${ }^{4}$.

A través de resonancias magnéticas realizadas a mujeres primerizas antes y después del embarazo, Hoekzema et al. ${ }^{4}$ mostraron una reducción simétrica en el volumen de la sustancia gris en la línea media cortical anterior y posterior, así como en secciones específicas de la corteza prefrontal y temporal. Estas zonas conforman un mapa que coincide, en gran medida, con una red que se asocia a la teoría de la mente -la habilidad para comprender y predecir la conducta de otras personas, sus conocimientos, sus intenciones y sus creencias ${ }^{54}$. Los cambios cerebrales evidenciaron que la pérdida de materia gris podría representar un ajuste fino de las sinapsis en redes neuronales más eficientes, es decir, una especialización cerebral.

Hoekzema et al. ${ }^{4}$, afirmaron que los cambios en el cerebro de las embarazadas y puérperas, si bien implican una disminución de materia gris, no necesariamente implican actividad cerebral reducida. Esto tiene relación con la comparación de procesos cerebrales en la pubertad, período en que también hay incrementos hormonales significativos. En la pubertad, las conexiones cerebrales más débiles son eliminadas, dejando una red neuronal más eficiente y especializada.

Las investigaciones en la permanencia de los cambios de cerebro durante los periodos reproductivos sugieren que algunos cambios podrían ser transitorios y retornar a los niveles previos al embarazo, mientras que otros podrían ser más permanentes ${ }^{43,55-58}$.

\section{Paridad y cambios cognitivos}

El perfil hormonal característico del embarazo y el postparto, necesario para inducir la conducta materna, interviene en muchos de los efectos cognitivos observados con la experiencia reproductiva. Los perfiles hormonales durante y después del embarazo también son alterados por la experiencia reproductiva previa $^{3}$. Pese a que aún hay poca información disponible referida a las diferencias entre mujeres primerizas y múltiples, se ha hipotetizado sobre el efecto de paridad sobre los cambios neuropsicológicos². En este sentido, Anderson y Rutherfood ${ }^{13}$ hallaron un menor rendimiento en tareas de memoria en mujeres primíparas respecto a las secundíparas o multíparas.

Dados los cambios hormonales de la mujer a largo plazo después del primer embarazo, Parsons et al..$^{28}$ investigaron si la historia reproductiva afectaba el rendimiento cognitivo de las mujeres de manera diferente en una batería de pruebas neuropsicológicas durante el embarazo y después del parto. Como resultado se obtuvo que los déficits cognitivos informados durante el embarazo no estaban relacionados con la historia previa de embarazo; todas las mujeres presentaban un peor desempeño de igual manera. Sin embargo, después del parto, las primíparas obtuvieron puntuaciones significativamente menores que las multíparas en todas las medidas de los test neuropsicológicos. Con estos hallazgos, y aun controlando la perturbación del estado de ánimo, hipotetizaron que las primíparas experimentaron mayor estrés y ansiedad asociados con el parto y el cuidado infantil respecto a las mujeres multíparas, lo que resultó en una mayor distracción y un menor rendimiento en las pruebas neuropsicológicas después del parto, especialmente la memoria verbal (capacidad de aprender y recordar nuevas palabras).

Las múltiples experiencias reproductivas llevan a niveles más altos de estradiol y a niveles más bajos de prolactina, en comparación con las mujeres embarazadas primíparas ${ }^{3,59}$. Las diferencias en los niveles hormonales según la paridad también pueden afectar la memoria espacial. En este sentido, murinos multigravidas superaron a las primigrávidas en la tarea de colocación de objetos en los días 2 a 17 de gestación ${ }^{59}$. Más aún, Workman et al. ${ }^{3}$ concluyeron que los diferentes tipos de memoria pueden estar influenciados por el embarazo y los efectos pueden depender del día gestacional, de los niveles hormonales fluctuantes y de la experiencia reproductiva previa. Por otro lado, Gly$\mathrm{nn}^{11}$ encontró que la memoria verbal disminuida en el embarazo y el postparto fue más severa con el incremento de la paridad, donde madres multigravidas se desempeñaron de manera menor que las bigrávidas, que a su vez se desempeñaron peor que las primigrávidas. Este hallazgo provee una indicación de que el efecto de reproducción en la estructura cerebral y la función es acumulativo y duradero. Los mecanismos de estos efectos no son conocidos, pero los niveles hormonales probablemente juegan un rol importante, ya que éstos difieren con la paridad ${ }^{3,59}$. Por ejemplo, murinos multíparas tienen niveles más altos de estradiol y niveles 
más bajos de prolactina a lo largo de la gestación comparada con ratas primíparas.

Así como hay estudios en donde se comparó el rendimiento de las funciones cognitivas en puérperas según la paridad (primíparas o multíparas), también se han llevado a cabo estudios en donde se comparó el rendimiento de las funciones cognitivas en mujeres que cursaban distintos periodos del embarazo y otras que cursaban el período postparto.

Respecto anterior, estudios prospectivos apoyan la idea de cambios en las funciones cognitivas durante el embarazo y postparto, pero los autores no han podido especificar con exactitud en qué momento ocurre la disminución en el rendimiento de las diferentes funciones cognitivas. Se pudo evidenciar que existen dificultades en tareas de aprendizaje de listas de palabras durante los diferentes trimestres del embarazo y postparto ${ }^{15,16,28}$; aunque en otros estudios las pruebas de memoria, planeamiento y de velocidad cognitiva fueron halladas peores únicamente en el tercer trimestre ${ }^{2,12}$.

La memoria verbal y atención en las puérperas están afectadas en el postparto inmediato ${ }^{2,11}$. Sin embargo, también es posible que, cuando se enfrentan a diferentes tareas, por ejemplo, aquellas que son más directamente relevantes para el cuidado de los hijos, como la multitarea, el rendimiento bajo estrés o la sensibilidad a las señales infantiles, las madres pueden exhibir un rendimiento mejorado $^{11}$. Las funciones son nuevamente equivalentes a las no madres en unos 10 a 13 meses postparto ${ }^{22} y$ podrían incluso ser acrecentadas dos años después del posparto ${ }^{5}$.

Se ha evidenciado de manera multivariada la relación entre los fallos en la producción lingüística y la paridad, las características sociosanitarias y el insomnio ${ }^{60}$. La paridad se relaciona positivamente con la presencia perseveraciones e intrusiones lingüísticas. Por el contrario, la asociación con intrusiones en el test de Stroop fue inversa. Este último fallo presentó una asociación directa con la severidad del insomnio. Las intrusiones se correlacionaron inversamente con la paridad y los días de postparto. Más aún, durante el período de postparto y lactancia se evidencia un menor desempeño de las habilidades de acceso y evocación de palabras. Esta variación de las estrategias de organización de la producción verbal sugiere que las fluctuaciones del control ejecutivo y la memoria semántica se asocian a una reestructuración lingüística ${ }^{61}$.

\section{Reflexiones desde la salud colectiva}

Todos los cambios en la fisiología y biología desencadenan también cambios en las funciones cognitivas, el sueño, el humor y las emociones de la mujer, así como en su comportamiento. Esto guarda relación con la mirada expresada en la presentación de la Estrategia Mundial para la Salud de la Mujer, el Niño y el Adolescente realizada por el Secretario de Naciones Unidas 2016-2030, en el cual se explicitan las deudas que las salud tiene en este periodo de la mujer en cuanto a la accesibilidad y equidad a la calidad de los servicios de salud, como así también, la recopilación de datos sobre necesidades que al no ser percibidas o visibilizadas no serán reconocidas como tales y menos aún cubiertas en consecuencia ${ }^{62}$. Asimismo, esto es relevante para generar políticas públicas y de ordenamiento jurídico de referencia desde donde surgen nuevos paradigmas de atención maternal y neonatal adaptada a las expectativas, creencias y valores de necesidades y derechos de quienes reclaman los servicios de sa$\operatorname{lud}^{63}$. En otras palabras, reconocer las variaciones pueden parecer a priori reduccionistas, pero sin revelarlas se pierde la posibilidad de ser plausibles de políticas sanitarias en consecuencia.

La mujer se enfrenta en la maternidad a un desafío único en su vida, donde demuestra la capacidad de enfrentar a situaciones novedosas adaptándose a los cambios necesarios para lograrlo. Esta maternidad también se modifica por apreciaciones sociales desde donde el autocuidado de la madre se ha empoderado y demanda en servicios de salud su adecuada oferta ${ }^{64}$. Si bien el interés en la temática es creciente, la mayoría de los estudios nacionales, regionales e internacionales indican que la atención del posparto todavía se centra en el cuidado del recién nacido, sin abordar a la mujer puérpera de manera integral ${ }^{65}$, circunscribiendo la atención al aparato genital. Esto implica considerar a la mujer en edad reproductiva como un agente productivo a nivel social reconociendo sus derechos individuales implícitos $^{66}$, lo cual ha sido advertido en el campo de la Salud Colectiva: do Carmo Oliveira et al. ${ }^{67}$ reconocen la necesidad de invertir en mejorar la estructura organizativa de la atención posparto para mejorar la calidad.

Finalmente, para lograr una adecuada comprensión de estos fenómenos, se precisan nuevos estudios que evalúen las implicancias de estos cambios neuropsicológicos en la morbimortalidad materno-infantil, salud ocupacional, familiar y el potencial impacto económico. 


\section{Consideraciones finales y conclusión}

El estudio de las variaciones cognitivas en el puerperio ha cobrado mayor interés en los últimos años. Esto evidencia el abordaje holístico de la mujer puérpera, comprendiendo otros procesos de salud en esta etapa vital. Estos cambios tienen su origen en las modificaciones psíquicas, anatomofisiológicas, sociales y emocionales propias del puerperio. A medida que éste avanza, hay una mejora en el control de las funciones cognitivas, lo que podría deberse a un proceso adaptativo. Más aún, las experiencias obstétricas también tienen un efecto en el estado cognitivo, siendo paridad una variable que influye.

Los seres humanos evolucionaron bajo presiones evolutivas complejas en donde las habilidades sociales debieron ser críticas para proporcionar cuidados maternos adecuados y criar exi- tosamente hijos en un ambiente social complejo ${ }^{4}$. El sistema de la teoría de la mente se considera un componente central del cerebro parental humano. Entonces, las modificaciones cerebrales que subyacen a los procesos sociales pueden considerarse una ventaja adaptativa, por ejemplo, facilitando la capacidad de la mujer para reconocer las necesidades de su hijo, para decodificar los estímulos sociales que pueden señalar una amenaza potencial, o para promover el vínculo 4 .

Aunque las revisiones narrativas pueden derivar en sesgos de selección ${ }^{68,69}$, es necesario destacar que este tipo de análisis se considera esencial en el debate de determinadas temáticas, planteando cuestionamientos y colaborando para la actualización del conocimiento ${ }^{68}$. Para comprender otros factores con potencial modulador del estado cognitivo se deben diseñar nuevas investigaciones.

\section{Colaboradores}

E Carrizo, J Domini y RYJ Quezada participaron en la concepción, planificación, análisis, interpretación, redacción final y revisión crítica del manuscrito. SV Serra y EA Soria participaron en el análisis y discusión de los datos, escribiendo el manuscrito y en la adquisición del financiamiento. AR Miranda es el investigador principal, contribuyó al diseño y la coordinación general del estudio, análisis y discusión de datos, redacción, revisión crítica y envío del manuscrito. Todos los autores participaron en la redacción y aprobaron la versión final del manuscrito. 


\section{Agradecimientos}

El presente trabajo ha sido financiado por el Instituto Nacional del Cáncer, el Instituto Nacional de la Yerba Mate, la Agencia Nacional de Promoción Científica y Tecnológica. AR Miranda fue apoyado por beca doctoral Secretaría de Ciencia y Tecnología (UNC).

\section{Referencias}

1. Piccardi L, Verde P, Bianchini F, Morgagni F, Guariglia C, Strollo F, Tomao E. Deficits in visuo-spatial but not in topographical memory during pregnancy and the postpartum state in an expert military pilot: a case report. BMC Res Notes 2015; 7(1):524.

2. Henry J, Sherwin B. Hormones and cognitive functioning during late pregnancy and postpartum: A longitudinal study. Behav Neurosci 2012; 126(1):73-85.

3. Workman J, Barha C, Galea L. Endocrine substrates of cognitive and affective changes during pregnancy and postpartum. Behav Neurosci 2012; 126(1):54-72.

4. Hoekzema E, Barba-Müller E, Pozzobon C, Picado M, Lucco F, García-García D, Soliva JC, Tobenia A, Desco M, Crone EA, Ballesteros A, Carmona S, Vilarrolla O. Pregnancy leads to long-lasting changes in human brain structure. Nature Neurosci 2016; 20(2):287-296.

5. Shansky RM. Sex Differences in the Central Nervous System. London: Academic Press, 2015.

6. Barha CK, Galea LAM. Influence of different estrogens on neuroplasticity and cognition in the hippocampus. Biochim Biophys Acta 2010; 1800(10):10561067.

7. Duff S, Hampson E. A beneficial effect of estrongen on working memory in Postmenopausal women taking Hormone replacement therapy. Horm Behav 2000; 38(4):262-276.

8. Martinelli SS, Cavalli SB. Sustainable and healthy diet: A narrative review of challenges and perspectives. Cien Saude Colet 2019; 24(11):4251-4262.

9. Kim P, Leckman J, Mayes L, Feldman R, Wang X, Swain J. The plasticity of human maternal brain: Longitudinal changes in brain anatomy during the early postpartum period. Behav Neurosci 2010; 124(5):695700.

10. Logan D, Hill K, Jones R, Holt-Lunstad J, Larson M. How do memory and attention change with pregnancy and childbirth? A controlled longitudinal examination of neuropsychological functioning in pregnant and postpartum women. J Clin Exp Neuropsychol 2014; 36(5):528-539.

11. Glynn LM. Giving birth to a new brain: Hormone exposures of pregnancy influence human memory. Psychoneuroendocrinology 2010;35(8):1148-1155.

12. Christensen H, Leach L, Mackinnon A. Cognition in pregnancy and motherhood: prospective cohort study. Br J Psychiatry 2010; 196(2):126-132.

13. Anderson M, Rutherford M. Cognitive Reorganization during Pregnancy and the postpartum period: an evolutionary perspective. Evol Psychol 2012; 10(4):659-687.

14. Cuttler C, Graf P, Pawluski JL, Galea LAM. Everyday life memory deficits in pregnant women. Can J Exp Psychol 2011; 65(1):27-37.

15. Mickes L, Wixted JT, Shapiro A, Scarff JM. The effects of pregnancy on memory: Recall is worse but recognition is not. J Clin Exp Neuropsychol 2009; 31(6):754761.

16. de Groot RH, Vuurman E, Hornstra G, Jolles J. Differences in cognitive performance during pregnancy and early motherhood. Psychol Med 2006; 36(7):10231032.

17. De Groot RH, Adam JJ, Hornstra G. Selective attention deficits during human pregnancy. Neurosci Lett 2003; 340(1):21-24. 
18. Casey P, Huntsdale C, Angus G, Janes C. Memory in pregnancy. II: Implicit, incidental, explicit, semantic, short-term, working and prospective memory in primigravid, multigravid and postpartum women. J Psychosom Obstet Gynaecol 1999; 20(3):158-164.

19. Janes C, Casey P, Huntsdale C, Angus G. Memory in pregnancy. I: Subjective experiences and objective assessment of implicit, explicit and working memory in primigravid and primiparous women. J Psychosom Obstet Gynaecol 1999; 20(2):80-87.

20. Messinis L, Vlahou C, Tsapanos V, Tsapanos A, Spilioti D, Papathanasopoulos P. Neuropsychological functioning in postpartum depressed versus nondepressed females and nonpostpartum controls. J Clin Exp Neuropsychol 2010; 32(6):661-666.

21. Buckwalter JG, Buckwalter DK, Bluestein BW, Stanczyk FZ. Pregnancy and postpartum: changes in cognition and mood. Prog Brain Res 2001; 133:303-319.

22. Crawley RA, Dennison K, Carter C. Cognition in pregnancy and her first year postpartum. Psychol Psychother 2003; 76(1):69-84.

23. Brett M, Baxendale S. Motherhood and memory: a review. Psychoneuroendocrinology 2001; 26(4):339-362.

24. Numan M, Woodside B. Maternity: Neural mechanisms, motivational processes, and physiological adaptations. Behav Neurosci 2010; 124(6):715-741.

25. Numan M. Neural basis of maternal behavior in the rat. Psychoneuroendocrinology 2006; 13(2):47-62.

26. Scanlan VF, Byrnes EM, Bridges RS. Reproductive experience and activation of maternal memory. Behav Neurosci 2006; 120(3):676-686.

27. Stolzenberg D, Stevens J, Rissman E. Experience-facilitated improvements in pup retrieval; evidence for an epigenetic effect. Horm Behav 2012; 62(2):128-135.

28. Parsons T, Thompson E, Buckwalter D, Bluestein B, Stanczyk F, Buckwalter J. Pregnancy history and cognition during and after pregnancy. Int J Neurosci 2004; 114(9):1099-1110.

29. Hamson D, Wainwright S, Taylor J, Jones B, Watson N, Galea L. Androgens Increase Survival of Adult-Born Neurons in the Dentate Gyrus by an Androgen Receptor-Dependent Mechanism in Male Rats. Endocrinology 2013; 154(9):3294-3304.

30. Holmes MM, Wide JK, Galea LAM. Low levels of estradiol facilitate, whereas high levels of estradiol impair, working memory performance on the radial arm maze. Behav Neurosci 2002; 116(5):928-934.

31. Vanston CM, Watson NV. Selective and persistent effect of foetal sex on cognition in pregnant women. Neuroreport 2005; 16(7):779-782.

32. Siebra Soares C, Moraes de Almondes K. Sono e cognição: implicações da privação do sono para a percepção visual e visuoespacial. Psico 2012; 43(1):85-92.

33. Banks S, Dinges D. Behavioral and physiological consequences of sleep restriction. J Clin Sleep Med 2007; 3(5):519-527.

34. Goel N, Rao H, Durmer J, Dinges D. Neurocognitive Consequences of Sleep Deprivation. Semin Neurol 2009; 29(4):320-339.

35. Fischer FM, Moreno CR, Rotenberg L. Trabalho em turnos e noturno na sociedade 24 horas. São Paulo: Atheneu; 2004.
36. Okamura H, Tsuda A. Short sleeping time and psychobiological responses to acute stress. Int J Psychophysiol 2010; 78(3):209-214.

37. Alvarado V, Arroyo G, Castro G, Fuentes F, Marín J, Soto G, Zumbado M. Impacto que tiene la falta de sueño sobre las habilidades cognitivas de una población de estudiantes de medicina. Med Leg Costa Rica 2012; 29(2):19-38.

38. Nielson CA, Deegan EG, Hung AS, Nunes AJ. Potential effects of sleep deprivation on sensorimotor in tegration during quiet stance in young adults. WURJHNS 2010; 1(1):1-8.

39. Swain AM, Michael MA, Starr KW, Gorman L. A prospective study of sleep, mood and cognitive function in postpartum and nonpostpartum women. Obstet Gynecol 1997; 90(3):381-386.

40. Bei B, Coo S, Trinder J. Sleep and Mood during Pregnancy and the Postpartum Period. Sleep Med Clin 2015; 10(1):25-33.

41. McBean AL, Kinsey SG, Montgomery-Downs HE. Effects of a single night of postpartum sleep on childless women's daytime functioning. Physiol Behav 2016; 156:137-147.

42. Neumann ID. Brain mechanisms underlying emotional alterations in the peripartum period in rats. $D e-$ press Anxiety 2003; 17(3):111-121.

43. Oatridge A, Holdcroft A, Saeed N, Hajnal JV, Puri BK, Fusi L, Bydder GM. Change in brain size during and after pregnancy: Study in healthy women and women with preeclampsia. AJNR Am J Neuroradiol 2002; 23(1):19-26.

44. Leuner B, Gould E. Dendritic growth in medial prefrontal cortex and cognitive flexibility are enhanced during the postpartum period. J Neurosci 2010; 30(40):13499-13503.

45. Macbeth AH, Luine VN. Changes in anxiety and cognition due to reproductive experience: A review of data from rodent and human mothers. Neurosci Biobehav Rev 2010; 34(3):452-467.

46. Barha CK, Galea LAM. Influence of different estrogens on neuroplasticity and cognition in the hippocampus. Biochim Biophys Acta 2010; 1800(10):10561067.

47. Pawluski JL, Brummelte S, Barha CK, Crozier TM, Galea LAM. Effects of steroid hormones on neurogenesis in the hippocampus of the adult female rodent during the estrous cycle, pregnancy, lactation and aging. Front Neuroendocrinol 2009; 30(3):343-357.

48. Snell RS. Clinical Neuroanatomy (seventh ed.). Philadelphia: Lippincott Williams \& Wilkins; 2009.

49. Maren S. Neurobiology of Pavlovian fear conditioning. Annu Rev Neurosci 2001; 24(1):897-931.

50. Burgess N. Spatial cognition and the brain. Ann N Y Acad Sci 2008; 1124(1):77-97.

51. McEwen BS. Central effects of stress hormones in health and disease: Understanding the protective and damaging effects of stress and stress mediators. Eur J Pharmacol 2008; 583(2-3):174-185.

52. Pawluski JL, Galea LA. Reproductive experience alters hippocampal neurogenesis during the postpartum period in the dam. Neuroscience 2007; 149(1):53-67. 
53. Galea LAM, Ormerod BK, Sampath S, Kostaras X, Wilkie DM, Phelps MT. Spatial working memory and hippocampal size across pregnancy in rats. Horm Behav 2000; 37(1):86-95.

54. Tirapu-Ustárroz J, Pérez-Sayes G, Erekatxo-Bilbao M, Pelegrín-Valero C. ¿Qué es la teoría de la mente? Rev Neurol 2007; 44(8):479-489.

55. Bodensteiner KJ, Cain P, Ray AS, Hamula LA. Effects of pregnancy on spatial cognition in female Hooded Long-Evans rats. Horm Behav 2006; 49(3):303-314.

56. Love G, Torrey N, McNamara I, Morgan M, Banks M, Hester NW, Lambert KG. Maternal experience produces long-lasting behavioral modifications in the rat. Behav Neurosci 2005; 119(4):1084-1096.

57. Gatewood JD, Morgan MD, Eaton M, McNamara IM, Stevens LF, Macbeth AH, Kinsley CH . Motherhood mitigates aging-related decrements in learning and memory and positively affects brain aging in the rat. Brain Res Bull 2005; 66(2):91-98.

58. Keyser-Marcus L, Stafisso-Sandoz G, Gerecke K, Jasnow A, Nightingale L, Lambert KG, Gatewood J, Kinsley $\mathrm{CH}$. Alterations of medial preoptic area neurons following pregnancy and pregnancy-like steroidal treatment in the rat. Brain Res Bull 2001; 55(6):737745.

59. Paris JJ, Frye CA. Estrous cycle, pregnancy, and parity enhance performance of rats in object recognition or object placement tasks. Reproduction 2008; 136(1)105-115.

60. Carrizo E, Domini J, Quezada RYJ. Fallos en la producción verbal dirigida en función de la paridad, características sociosanitarias y severidad del insomnio en puérperas de Gran Córdoba, Argentina, durante el periodo 2016-2017 [tesis]. Córdoba: Facultad de Ciencias Médicas, Universidad Nacional de Córdoba.

61. Miranda AR, Cortez MV, Rivadero L, Carrizo E, Domini J, Quezada RYJ, Serra SV, Soria EA. Análisis cuantitativo y cualitativo de fluidez verbal semántica durante el período de lactancia. Rev Fac Cien Med Univ Nac Cordoba 2017; Córdoba: Córdoba: Universidad Nacional de Córdoba; 2017.
62. Naciones Unidas. Objetivos del Desarrollo Sostenible [Internet]. 2015. [citado 2018 Ago 01]. Disponible en: http://www.un.org/sustainabledevelopment/es/ health/

63. Uribe RE. Mortalidad Materna [Internet]. 2009 [citado 2018 Ago 01]. Disponible en: http://www.facmed. unam.mx/sms/temas/2009/01_ene_2k9.pdf

64. García-de-Diego JM, Serrano-del-Rosal R. Universalización, paridad y evolución de la demanda de anestesia epidural durante el parto en Andalucía. Gaceta Sanitaria, in press 2018.

65. Rother ET. Revisão sistemática X revisão narrativa. Acta Paul Enfer. 2007; 20:5-6.

66. Bermúdez GM, Maldonado JMO. Derecho Familiar del Trabajo y de la Seguridad Social. Lex Social Rev Derechos Sociales 2018, 8:136-164.

67. Oliveira DDC, Mandu ENT, Correa ACDP, Tomiyoshi JT, Teixeira RC. Organizational structure of postpartum care in Family Health Strategy. Escola Anna Nery 2013, 17:446-454.

68. Cordeiro AM, Oliveira GMd, Rentería JM, Guimarães CA. Revisão sistemática: uma revisão narrativa. Rev Colégio Bras Cirurgiões 2007; 34:428-431.

69. Baratieri T, Natal S. Actions of the postpartum program in the primary health care: An integrative review. Cien Saude Colet 2019; 24(11):4227-4238.

Artículo presentado en 07/06/2018

Aprobado en 05/11/2018

Versión final presentada en 07/11/2018 